\title{
Associations between sinus tachycardia and adverse cardiovascular outcomes and mortality in cancer patients
}

\author{
Mohamad Hemu', Caleb J. Chiang ${ }^{1}$, Parva K. Bhatt ${ }^{1}$, Aamir Ahmed ${ }^{1}$, Kyaw Zaw Hein ${ }^{1}$, Talal Mourad ${ }^{2}$, \\ Megan E. Randall ${ }^{1}$, Andres P. Palomo ${ }^{1}$, Jason B. Kramer ${ }^{1}$, Ibtihaj Fughhi ${ }^{3}$, Louis Fogg ${ }^{4}$, Philip Bonomi ${ }^{3}$, \\ Tochukwu M. Okwuosa ${ }^{5}$
}

${ }^{1}$ Department of Internal Medicine, Rush University Medical Center, Chicago, IL, USA; ${ }^{2}$ College of Medicine, University of Illinois, Chicago, IL, USA; ${ }^{3}$ Department of Oncology, Rush University Medical Center, Chicago, IL, USA; ${ }^{4}$ Department of Community, Systems and Mental Health Nursing, College of Nursing, Rush University Medical Center, Chicago, IL, USA; ${ }^{5}$ Director of Cardio-Oncology Services, Department of Cardiology, Rush University Medical Center, Chicago, IL, USA

Contributions: (I) Conception and design: M Hemu, PK Bhatt, A Ahmed, TM Okwuosa; (II) Administrative support: TM Okwuosa, PK Bhatt, L Fogg; (III) Provision of study materials or patients: M Hemu, PK Bhatt, TM Okwuosa; (IV) Collection and assembly of data: M Hemu, CJ Chiang, P Bonomi, A Ahmed, KZ Hein, T Mourad, ME Randall, AP Palomo, JB Kramer, I Fughhi; (V) Data analysis and interpretation: M Hemu, CJ Chiang, L Fogg, P Bonomi, TM Okwuosa; (VI) Manuscript writing: All authors; (VII) Final approval of manuscript: All authors.

Correspondence to: Tochi M. Okwuosa, DO, FACC, FAHA. Associate Professor of Medicine and Cardiology, Director, Cardio-Oncology Program, Rush University Medical Center I 1717 West Congress Parkway, Kellogg Bldg., Suite 328 । Chicago, IL 60612, USA. Email: tokwuosa@rush.edu.

\begin{abstract}
Background: Sinus tachycardia in cancer reflects a significant multi-system organ stressor and disease, with sparse literature describing its clinical significance. We assessed cardiovascular (CV) and mortality prognostic implications of sinus tachycardia in cancer patients.

Methods: We conducted a case-control study of 622 cancer patients at a U.S. urban medical center from 2008 to 2016. Cases had ECG-confirmed sinus tachycardia [heart rate (HR) $\geq 100 \mathrm{bpm}$ ] in $\geq 3$ different clinic visits within 1 year of cancer diagnosis excluding a history of pulmonary embolism, thyroid dysfunction, left ventricular ejection fraction $<50 \%$, atrial fibrillation/flutter, HR $>180 \mathrm{bpm}$. Adverse CV outcomes (ACVO) were heart failure with preserved ejection fraction (HFpEF), HF with reduced EF (HFrEF), hospital admissions for HF exacerbation (AHFE), acute coronary syndrome (ACS). Regression analyses were conducted to examine the effect of sinus tachycardia on overall ACVO and survival.

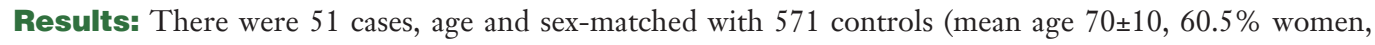
$76.4 \%$ Caucasian). In multivariate analysis over a 10-year follow-up period, sinus tachycardia (HR $\geq 100 v s$. $<100 \mathrm{bpm}$ ) was an independent predictor of overall ACVO (OR 2.8, 95\% CI: 1.4-5.5; P=0.006). There was increased incidence of HFrEF (OR 3.3, 95\% CI: 1.6-6.5; P=0.004) and AHFE (OR 6.3, 95\% CI: 1.6-28; $\mathrm{P}=0.023)$, but not HFpEF or ACS $(\mathrm{P}>0.05)$ compared with controls. Sinus tachycardia was a significant predictor of overall mortality after adjusting for significant covariates (HR 2.9, 95\% CI 1.8-5; $\mathrm{P}<0.001$ ).

Conclusions: Independent of typical factors that affect cardiovascular disease, sinus tachycardia around the time of cancer treatment is associated with increased ACVO and mortality in cancer patients at 10 years of follow-up.
\end{abstract}

Keywords: Sinus tachycardia; cancer survivorship; risk prediction; heart failure; outcomes

Submitted May 06, 2021. Accepted for publication Jul 14, 2021.

doi: $10.21037 /$ jtd-21-779

View this article at: https://dx.doi.org/10.21037/jtd-21-779 


\section{Introduction}

Numerous studies have examined elevated resting heart rate (RHR) as an independent risk factor for mortality $(1,2)$. These studies have demonstrated that elevated RHR has significant associations with cardiovascular disease, diabetes mellitus, stroke, renal disease, and all-cause mortality (3-6). More recently, there has been heightened interest in the possible contributions of cardiovascular disease to the elevated mortality observed in patients with various forms of advanced cancer. Prior studies have demonstrated increased stress on cardiac function in cancer patients due to higher cardiac output and maximal rate of pressure rise in isovolumetric contraction compared to healthy subjects (7). von Haelhing et al. (7) demonstrated that patients with cancer tended to have altered cardiac hemodynamics, including elevated blood pressure, cardiac output, and stroke volume compared to controls and patients with chronic heart failure. Thus, researchers hypothesize that these alterations in cardiac hemodynamics lead to adverse cardiac events, such as blood pressure dysregulation, ischemia, arrhythmias, and heart failure. Recent studies have examined the prognostic value of elevated RHR in patients with cancer (8), but none to our knowledge have investigated the prognostic value of ECG-confirmed sinus tachycardia defined as heart rate $(\mathrm{HR}) \geq 100$ beats per minute (bpm) in this patient population. Sinus tachycardia in cancer may reflect a significant multi-system organ stressor and disease, with sparse literature describing its clinical significance.

The goal of this analysis is to determine associations between baseline sinus tachycardia and future risk of adverse cardiovascular outcomes (ACVO) and increased mortality in cancer patients.

We present the following article in accordance with the STROBE reporting checklist (available at https://dx.doi. org/10.21037/jtd-21-779).

\section{Methods}

\section{Study population and patient selection}

This case-control study was conducted at a U.S. urban academic center. The study was reviewed and approved by the Institutional Review Board (15061101-IRB01), and individual consent for this retrospective analysis was waived. This study was conducted in accordance with the Declaration of Helsinki (as revised in 2013). Patients presented to the clinics with newly diagnosed malignancy between 2008 and 2016. Only patients with histopathological confirmation of cancer diagnosis and documented sinus tachycardia $(H R \geq 100)$ on $\geq 3$ consecutive clinic visits within 1 year of cancer diagnosis were included in the study. Patients were assessed in clinic on a weekly to monthly basis. All included patients were followed at this medical center for the duration of the study. Exclusion criteria included: (I) age $<18$ years old; (II) active treatment for venous thromboembolism (VTE); (III) untreated thyroid disease; (IV) known heart failure with reduced ejection fraction $(<50 \%)$; (V) clinical signs of decompensated heart failure; (VI) documented history of atrial fibrillation or flutter; and (VII) patients with a heart rate $(\mathrm{HR})>180 \mathrm{bpm}$. Individuals on active treatment for VTE (including pulmonary embolism) were excluded, as the presence of VTE was viewed as a potential confounder for sinus tachycardia.

The diagnosis of sinus tachycardia was confirmed by reviewing serial 12-lead electrocardiogram (ECG) data obtained following the initial diagnosis of cancer and during consecutive visits to the oncology clinics. The study variables (Table 1) were extracted from the electronic health records and stored in the REDCap database software. Data regarding survival information and outcomes were obtained directly from the patients' charts. Additionally, the medical center had access to the national death index to further evaluate survival outcomes. Data were collected and reviewed by two independent research coordinators; then exported to SPSS software for statistical analysis.

The primary outcomes of interest for ACVO were newonset heart failure, including heart failure with reduced ejection fraction (HFrEF; EF $\leq 40 \%$ ), new-onset heart failure with preserved ejection fraction (HFpEF; $\mathrm{EF} \geq 50 \%$ ), admissions for heart failure exacerbation (AHFE; both HFrEF and HFpEF) and acute coronary syndromes (ACS). We also assessed the implications of sinus tachycardia on overall survival up to 10 years from the date of an initial cancer diagnosis.

\section{Statistical analyses}

All calculations were performed using SPSS/PC statistical program (version 21, SPSS Inc., Chicago, IL, USA). Continuous variables were reported as means \pm SD while categorical variables were expressed as numbers or ratios. Cases and controls were matched by age and sex. Controls to cases were matched 1:10 to increase the power of the study. Groups were compared using unpaired Student's 
Table 1 Baseline characteristics of patients according to baseline resting heart rate

\begin{tabular}{|c|c|c|c|c|}
\hline Variables & All patients & $H R \leq 100$ & $\mathrm{HR}>100$ & $P$ value \\
\hline Age & $70 \pm 10$ & $71 \pm 9$ & $67 \pm 13$ & 0.674 \\
\hline Gender (female \%) & $376(60.5)$ & $348(60.9)$ & $28(54.9)$ & 0.502 \\
\hline Race (white \%) & $475(76.4)$ & 445 (77.9) & $30(58.8)$ & 0.005 \\
\hline AJCC (Stage 4) & $297(47.8)$ & 279 (48.9) & 18 (35.3) & 0.375 \\
\hline Albumin & $3.44 \pm 1.47$ & $3.5 \pm 1.5$ & $3 \pm 0.89$ & 0.043 \\
\hline Hemoglobin & $11.6 \pm 2.4$ & $11.7 \pm 2.2$ & $10.5 \pm 2.8$ & 0.001 \\
\hline$N / L$ & $5.3 \pm 7.43$ & $5.21 \pm 7.5$ & $5.7 \pm 7.44$ & 0.094 \\
\hline Hyperlipidemia & $230(37.0)$ & $210(36.8)$ & $20(39.2)$ & 0.651 \\
\hline Coronary artery disease & $91(14.6)$ & $83(14.5)$ & $8(16.0)$ & 0.775 \\
\hline Stroke/TIA & $57(9.2)$ & $54(9.5)$ & $3(6.0)$ & 0.419 \\
\hline Chronic kidney disease & $121(19.5)$ & $98(17.1)$ & $23(45.1)$ & $<0.001$ \\
\hline Leukocytosis & $157(25.2)$ & $141(24.7)$ & $16(31.3)$ & 0.251 \\
\hline Smoking & $298(47.9)$ & $273(47.8)$ & $25(49.0)$ & 0.758 \\
\hline Beta-blockers & $204(32.8)$ & $180(31.5)$ & $24(47.0)$ & 0.017 \\
\hline Spironolactone & $8(1.3)$ & $7(1.2)$ & $1(2.0)$ & 0.640 \\
\hline \multicolumn{5}{|l|}{ Type of malignancy } \\
\hline Primary lung & $272(43.7)$ & $257(45.0)$ & $15(29.0)$ & 0.001 \\
\hline Lymphoma & $130(21.0)$ & $120(21.0)$ & $10(19.6)$ & \\
\hline Multiple myeloma & $72(11.6)$ & $69(12.1)$ & $3(6.0)$ & \\
\hline Leukemia & $108(17.4)$ & $88(15.4)$ & $20(39.2)$ & \\
\hline Other & $40(6.4)$ & $37(6.5)$ & $3(6.0)$ & \\
\hline Chemotherapy at time of visit & $341(54.8)$ & $314(55.0)$ & $27(52.9)$ & 0.725 \\
\hline Anthracyclines & $118(19.0)$ & $107(18.7)$ & $11(22.0)$ & 0.573 \\
\hline Radiation & $267(43.0)$ & $249(43.6)$ & $18(35.3)$ & 0.297 \\
\hline
\end{tabular}

Data presented as mean \pm standard deviation for continuous variables and $\mathrm{n}(\%)$ for categorical variables. BMI, body mass index; AJCC, American Joint Committee on Cancer; N/L, neutrophil/lymphocyte ratio; ACE-I/ARB, angiotensin-converting enzyme inhibitors/angiotensin receptor blockers, radiation included therapies delivered to the chest and mediastinum. 
Table 2 Univariate and multivariate logistics regression analysis for predictors of adverse cardiovascular outcomes

\begin{tabular}{|c|c|c|c|c|}
\hline Variables & \multicolumn{2}{|c|}{ Univariate } & \multicolumn{2}{|c|}{ Multivariate } \\
\hline Sinus Tachycardia & $3.8(2.1-6.6)$ & 0.001 & $3.1(1.5-6.2)$ & 0.002 \\
\hline Race (white \%) & $1.9(1.2-2.8)$ & 0.003 & $1.8(1.1-2.9)$ & 0.017 \\
\hline Albumin & $0.64(0.48-0.86)$ & 0.003 & $0.88(6.3-1.2)$ & 0.465 \\
\hline Beta-Blockers & $0.31(0.21-0.45)$ & 0.001 & $0.246(0.25-0.62)$ & 0.001 \\
\hline Renal disease (GFR $<60 \mathrm{~mL} / \mathrm{min} / 1.73 \mathrm{~m}^{2}$ ) & $1.4(1.36-2.7)$ & 0.010 & $0.730(0.43-1.24)$ & 0.246 \\
\hline
\end{tabular}

Covariates with $\mathrm{P}<0.05$ in Table 1 (race, albumin, hemoglobin, beta-blocker use, chronic kidney disease), anticoagulation, type of malignancy) were included in multivariate analysis. OR, Odds ratio; $\mathrm{Cl}$, confidence interval. GFR, glomerulus filtration rate.

$t$-test for continuous variables while $\chi^{2}$ was used to evaluate dichotomous variables.

Univariate analyses for predictors of ACVO were conducted. In addition, odds ratios were calculated using a multivariate logistic regression model which included sinus tachycardia in addition to significant covariates and clinically relevant predictors of ACVO (age, race, beta-blocker use, chronic kidney disease, type of malignancy, albumin, hemoglobin, anticoagulation, anthracyclines, and radiation).

Univariate Cox-proportional hazards analyses were conducted to identify predictors of survival in patients with cancer. Multivariate survival analysis was performed to further investigate the impact of sinus tachycardia on overall survival over a 10 -year follow-up period. In our first model, we included all statistically significant predictors of mortality plus covariates that were significantly different between the sinus tachycardia group and patients with HR $<100$ : sinus tachycardia, race, hemoglobin, albumin, betablocker use, chronic kidney disease, type of malignancy, and use of anticoagulation (model 1). To eliminate any possible confounders, our second model (model 2) included sinus tachycardia, age, and gender in addition to clinically relevant predictors of mortality [smoking, history of coronary artery disease (CAD), diabetes, aspirin, betablockers, history of stroke, radiation and anthracyclines].

$\mathrm{P}$ value $<0.05$ was considered statistically significant in all analyses.

\section{Results}

\section{Study population}

We included 51 patients with ECG-identified baseline sinus tachycardia (HR $\geq 100 \mathrm{bpm}$ ), and 571 age and sex-matched controls with $\mathrm{HR}<100 \mathrm{bpm}$ (total 622 patients; mean age $70 \pm 10$ years, $60.5 \%$ women, $76.4 \%$ Caucasian). Baseline characteristics were stratified according to heart rate and the total cohort are summarized in Table 1. Of the 622 patients, $43.7 \%$ had primary lung cancer, $21 \%$ had lymphoma, $11.6 \%$ had multiple myeloma, $17.4 \%$ had leukemia, and $6.4 \%$ had either breast, thyroid, neuroendocrine, colorectal, or pancreatic malignancies. A total of $47.8 \%$ of patients were classified as stage 4 cancer according to the American Joint Committee on Cancer (AJCC). The stage of cancer was not significantly different between the two groups [18 (35.3) vs. 279 (48.9), $\mathrm{P}=0.375]$. Significantly more Caucasian patients were in the HR <100 bpm group [445 (77.9) vs. 30 (58.8), $\mathrm{P}=0.005]$. Cardiovascular risk factors including age, gender, body mass index (BMI), diabetes, hypertension, $\mathrm{CAD}$, and smoking status were not significantly different between the study group and controls. Sinus tachycardia patients were noted to have overall lower levels of serum albumin ( 3 vs. $3.5 \mathrm{~g} / \mathrm{dL}, \mathrm{P}=0.043)$ and hemoglobin (10.5 vs. $11.7 \mathrm{~g} / \mathrm{dL}, \mathrm{P}=0.001$ ), compared with controls. Additionally, underlying renal dysfunction (GFR $<60$ $\mathrm{mL} / \mathrm{min} / 1.73 \mathrm{~m}^{2}$ ) was more prevalent in the sinus tachycardia group (45.1\% vs. $17.1 \%, \mathrm{P}<0.001)$.

\section{Predictors of overall adverse cardiovascular outcomes}

In univariate analysis (Table 2), over a follow-up period of 10 years, sinus tachycardia was a predictor of overall ACVO (OR 3.8, 95\% CI: 2.1-6.6; P=0.001). Race, albumin, hemoglobin, beta-blocker use, and chronic kidney disease were also identified as significant predictors of overall ACVO. In multivariate analysis, sinus tachycardia remained an independent predictor of ACVO (OR 3.1, 95\% CI: 1.56.2; $\mathrm{P}=0.002)$ even after adjusting for significant covariates 


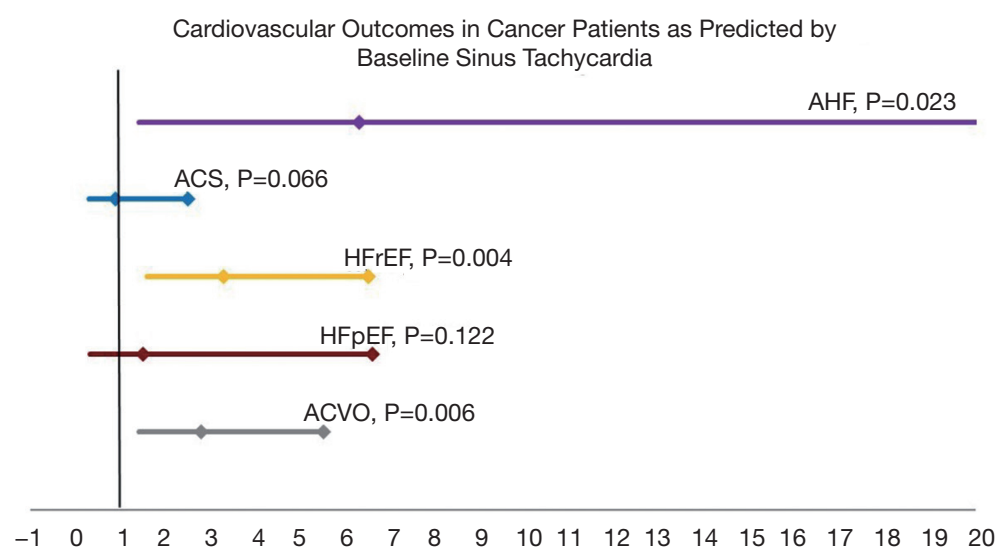

Figure 1 Cardiovascular Outcomes as Predicted by Baseline Sinus Tachycardia. Legend: Odds Ratio (OR), 95\% Confidence Intervals; for heart rates $\geq 100 v s$. $<100 \mathrm{bpm}$. Sinus tachycardia was associated with increased incidence of AHFE and HFrEF, but not HFpEF or ACS. ACS, Acute Coronary Syndromes; AHFE, Acute Heart Failure Exacerbation; HFpEF, Heart Failure with preserved Ejection Fraction; HFrEF, Heart Failure with reduced Ejection Fraction; CVO, Cardiovascular Outcomes. Model adjusted for age, race, beta-blocker use, renal disease $\left(\mathrm{GFR}<60 \mathrm{~mL} / \mathrm{min} / 1.73 \mathrm{~m}^{2}\right.$ ), type of malignancy, albumin, hemoglobin, anticoagulation, anthracyclines, and radiation

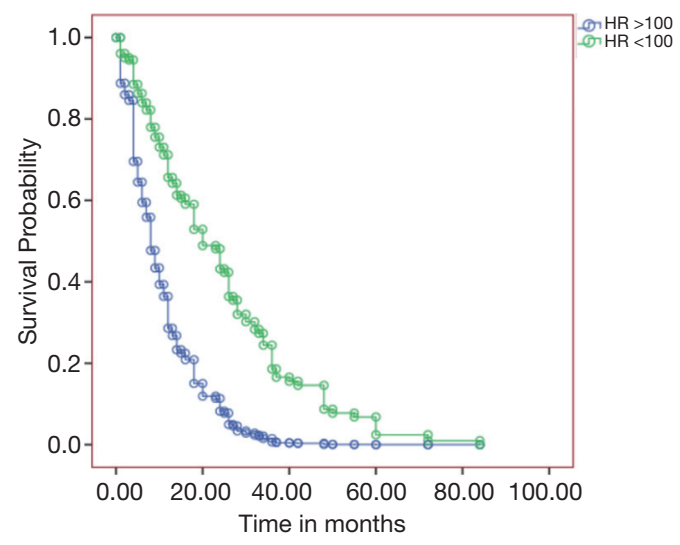

Figure 2 Kaplan-Meier survival curve in cancer patients as predicted by resting heart rate. Sinus tachycardia was a significant predictor of mortality (HR 2.9, 95\% CI: 1.8-5; $\mathrm{P}<0.001$ ) after adjusting for variables in model 1 (race, albumin, hemoglobin, beta-blockers, chronic kidney disease (GFR $<60 \mathrm{~mL} / \mathrm{min}$ ), anticoagulation and type of malignancy).

(Table 2). In a separate multivariate logistic regression model, sinus tachycardia remained an independent predictor of ACVO after adjusting for clinically and statistically significant covariates (OR 2.8, 95\% CI: 1.4-5.5; $\mathrm{P}=0.006$ ) (Figure 1). As depicted in Figure 1, patients with sinus tachycardia had increased incidence of HFrEF (OR 3.3, 95\% CI: 1.6-6.5; $\mathrm{P}=0.004)$ and acute heart failure exacerbation (AHFE) [OR 6.3, 95\% CI: 1.6-28; P=0.023]; but not HFpEF or ACS $(\mathrm{P}>0.05)$ as compared to controls.

\section{Predictors of overall survival}

We used univariable Cox-proportional hazards analyses, which identified sinus tachycardia (HR 2.4, 95\% CI: $1.5-$ 3.6; $\mathrm{P}<0.001)$ and serum hemoglobin $\leq 10 \mathrm{~g} / \mathrm{dL}$ (HR 1.8, 95\% CI: 1.26-2.6) as significant predictors of mortality in patients with cancer (data not shown). To further investigate the impact of sinus tachycardia on survival in patients with cancer, we performed multivariable survival analyses with Kaplan Meier survival curves shown in Figure 2. Sinus tachycardia remained an independent predictor of survival after adjusting for all covariates in model 1 which included serum hemoglobin (HR 2.9, $95 \%$ CI: $1.8-5$; $\mathrm{P}<0.001$ ) (Figure 2). Additionally, sinus tachycardia remained an independent predictor of mortality in patients with cancer even after adjusting for all covariates in model 2, including smoking, CAD, diabetes, aspirin, beta-blockers, history of stroke, radiation and anthracyclines (HR 2.5, 95\% CI: $1.5-4 ; \mathrm{P}=0.001)$.

\section{Discussion}

We sought to address the clinical significance and prognostic value of sinus tachycardia in patients with cancer. This study shows that sinus tachycardia around the time of cancer treatment, independent of typical risk 
factors that affect patients with cancer, was associated with the development of ACVO and a higher risk of all-cause mortality at 10 years of follow-up. To our knowledge, this is the first study to determine the prognostic impact of sinus tachycardia on cardiovascular outcomes and mortality in cancer patients.

Many patients with cancer undergoing chemo- and/or chest radiation therapy are at increased risk of cardiovascular disease. It has been well documented that certain types of chemotherapy such as anthracyclines, tyrosine kinase inhibitors, microtubule inhibitors, and antimetabolites are associated with adverse effects on the cardiovascular system such as the development of heart failure, ischemia, or arrhythmias (9-14). Additionally, radiation therapy is associated with increased incidence of myocardial fibrosis, valvular disease, cardiomyopathy, conduction abnormalities, and CAD (15-17). High cardiac output states are frequently noted in patients with underlying cancer (18). The pathophysiology of high output states in this patient population is often related to underlying anemia, hyperthyroidism, shunting of blood through the tumors, or secretion of antidiuretic hormone (19). In patients undergoing therapy with multiple chemotherapeutic agents and radiation, these negative cardiovascular effects could be amplified $(20,21)$. As a result, identifying patients with a higher risk for cardiotoxicity is a key strategy in reducing morbidity and mortality associated with cancer treatment.

The effects of elevated RHR at baseline on cardiovascular mortality and morbidity in patients with cancer have been extensively studied and well documented in the literature (1-6). Elevated RHR such as sinus tachycardia results in decreased diastolic filling, decreased myocardial perfusion, and decreased left ventricular function. Over time, these effects may result in myocardial damage and congestive heart failure $(22,23)$. Similarly in the Framingham study, elevated RHR was directly associated with increased all-cause mortality in patients with baseline hypertension after 36 years of follow-up (2). Additionally, the prognostic effects of RHR on patients with CAD and left ventricular systolic dysfunction were studied in the randomized BEAUTIFUL (MorbidityMortality Evaluation of the I(f) Inhibitor Ivabradine in Patients with Coronary Disease and Left Ventricular Dysfunction) trial. The authors reported that patients with RHR $\geq 70$ bpm had more frequent cardiovascular events including an increased number of admissions for heart failure, myocardial infarction, and cardiovascular-related mortality during 19-month of follow-up (24). Given the substantial effects of HR on cardiovascular outcomes and survival, slowing the HR has been a target for the management of patients with $\mathrm{CAD}$ and heart failure.

Similarly, our study demonstrated that baseline sinus tachycardia resulted in a 3 -fold increased incidence of HFrEF, a 6-fold increase in AHFE, and an almost a 3-fold increase in mortality. This study, while uniquely focused on cancer patients, adds to the current evidence that elevated RHR is associated with increased mortality and morbidity. These findings raise the question of whether controlling a patient's HR with medication could improve mortality and decrease the risk of ACVO and mortality in this population. Beta-blockers and Ivabradine are two types of drugs that result in a substantial decrease in HR and increased survival in patients with underlying cardiovascular disease $(25,26)$. However, no randomized control trials have been performed to evaluate the potential benefits of these medications in treating patients with cancer and sinus tachycardia.

Sinus tachycardia in the setting of cancer may be caused by pulmonary thromboembolism disease (27), a side effect of cancer therapy $(14,17)$, or potentially dysautonomia (28). The presence of sinus tachycardia in these patients may be a compensatory mechanism (and therefore, a marker) reflecting their body's adjustment to systemic and multiorgan inflammatory and hypermetabolic states in the setting of their underlying malignancy. This idea, however, raises another question as to whether medically controlling the HR could harm these patients by blunting their cardiovascular response to the underlying disease process. Additionally, there is no strong evidence to support treating isolated sinus tachycardia in the absence of underlying cardiovascular disease. Therapeutic considerations for these patients are largely focused on lifestyle modification including regular aerobic exercise, hydration, avoiding stimulants, and maintaining normal body weight.

Some limitations of our study include the retrospective nature of the study and the small number of patients in the study group. We compensated for the latter concern by using the 1:10 matched control group to increase the power of our study to detect differences between both groups. Significantly less Caucasians were included in the $>100$ BPM group $(\mathrm{P}=0.005)$. This difference is likely related to the small sample size available. Given the study design, the authors were unable to account for selection bias and residual confounding. Furthermore, we could not determine if sinus tachycardia was present in these patients before their diagnosis of cancer because most of them were referred to our oncology clinic after a cancer diagnosis. Additionally, we were not able to determine the exact cause 
of death in these patients. This is partly because the clinical picture in advanced cancer is rather complex and varies greatly between cancer types. We should note that the majority of the patients included in this study were elderly ( $70 \pm 10$ years). Additional research is required to evaluate the risk of adverse cardiac outcomes and mortality in a younger patient population.

\section{Conclusions}

Independent of typical factors that affect disease, baseline sinus tachycardia around the time of cancer treatment was associated with ACVO and increased mortality at 10 years of follow-up. Future studies are required to assess the presence of sinus tachycardia as a marker of systemic inflammation and overall disease prognosis in the setting of cancer. Moreover, further research is needed to evaluate the impact of treating sinus tachycardia using agents such as beta-blockers or ivabradine on the risk reduction of ACVO and improvement in overall mortality.

\section{Acknowledgments}

This work was presented in The American College of Cardiology $68^{\text {th }}$ Annual Scientific Sessions. The primary author of this manuscript holds the copyright to reuse the material presented in the following abstract: "Elevated Resting Heart Rate Improves Risk Stratification For Adverse Cardiovascular Outcomes in Cancer Patients. Fournal of the American College of Cardiology Mar 2019, 73 (9 Supplement 1) 1765; DOI: 10.1016/S0735-1097(19)32371-X".

Funding: This work was supported solely by departmental funding.

\section{Footnote}

Reporting Checklist: The authors have completed the STROBE reporting checklist. Available at https://dx.doi. org/10.21037/jtd-21-779

Data Sharing Statement: Available at https://dx.doi. org/10.21037/jtd-21-779

Peer Review File: Available at https://dx.doi.org/10.21037/ jtd-21-779

Conflicts of Interest: All authors have completed the ICMJE uniform disclosure form (available at https://dx.doi. org/10.21037/jtd-21-779). The authors have no conflicts of interest to declare.

Ethical Statement: The authors are accountable for all aspects of the work in ensuring that questions related to the accuracy or integrity of any part of the work are appropriately investigated and resolved. The study was reviewed and approved by the Institutional Review Board (15061101IRB01), and individual consent for this retrospective analysis was waived. This study was conducted in accordance with the Declaration of Helsinki (as revised in 2013).

Open Access Statement: This is an Open Access article distributed in accordance with the Creative Commons Attribution-NonCommercial-NoDerivs 4.0 International License (CC BY-NC-ND 4.0), which permits the noncommercial replication and distribution of the article with the strict proviso that no changes or edits are made and the original work is properly cited (including links to both the formal publication through the relevant DOI and the license). See: https://creativecommons.org/licenses/by-nc-nd/4.0/.

\section{References}

1. Mensink GB, Hoffmeister $H$. The relationship between resting heart rate and all-cause, cardiovascular and cancer mortality. Eur Heart J 1997;18:1404-10.

2. Kannel WB, Kannel C, Paffenbarger RS Jr, et al. Heart rate and cardiovascular mortality: the Framingham Study. Am Heart J 1987;113:1489-94.

3. Lonn EM, Rambihar S, Gao P, et al. Heart rate is associated with increased risk of major cardiovascular events, cardiovascular and all-cause death in patients with stable chronic cardiovascular disease: an analysis of ONTARGET/TRANSCEND. Clin Res Cardiol 2014;103:149-59.

4. Palatini P, Casiglia E, Pauletto P, et al. Relationship of tachycardia with high blood pressure and metabolic abnormalities: a study with mixture analysis in three populations. Hypertension 1997;30:1267-73.

5. Böhm M, Schumacher H, Schmieder RE, et al. Resting heart rate is associated with renal disease outcomes in patients with vascular disease: results of the ONTARGET and TRANSCEND studies. J Intern Med 2015;278:38-49.

6. Wannamethee G, Shaper AG. The association between heart rate and blood pressure, blood lipids and other cardiovascular risk factors. J Cardiovasc Risk 1994;1:223-30. 7. von Haehling S, Lainscak M, Kung T, et al. Non-invasive 
assessment of cardiac hemodynamics in patients with advanced cancer and with chronic heart failure: a pilot feasibility study. Arch Med Sci 2013;9:261-7.

8. Anker MS, Ebner N, Hildebrandt B, et al. Resting heart rate is an independent predictor of death in patients with colorectal, pancreatic, and non-small cell lung cancer: results of a prospective cardiovascular long-term study. Eur J Heart Fail 2016;18:1524-34.

9. Swain SM, Whaley FS, Ewer MS. Congestive heart failure in patients treated with doxorubicin: a retrospective analysis of three trials. Cancer 2003;97:2869-79.

10. Ewer SM, Ewer MS. Cardiotoxicity profile of trastuzumab. Drug Saf 2008;31:459-67.

11. Tocchetti CG, Gallucci G, Coppola C, et al. The emerging issue of cardiac dysfunction induced by antineoplastic angiogenesis inhibitors. Eur J Heart Fail 2013;15:482-9.

12. Force T, Krause DS, Van Etten RA. Molecular mechanisms of cardiotoxicity of tyrosine kinase inhibition. Nat Rev Cancer 2007;7:332-44.

13. Seidman A, Hudis C, Pierri MK, et al. Cardiac dysfunction in the trastuzumab clinical trials experience. J Clin Oncol 2002;20:1215-21.

14. Albini A, Pennesi G, Donatelli F, et al. Cardiotoxicity of anticancer drugs: the need for cardio-oncology and cardiooncological prevention. J Natl Cancer Inst 2010;102:14-25.

15. Darby SC, McGale P, Taylor CW, et al. Long-term mortality from heart disease and lung cancer after radiotherapy for early breast cancer: prospective cohort study of about 300,000 women in US SEER cancer registries. Lancet Oncol 2005;6:557-65.

16. Lancellotti P, Nkomo VT, Badano LP, et al. Expert consensus for multi-modality imaging evaluation of cardiovascular complications of radiotherapy in adults: a report from the European Association of Cardiovascular Imaging and the American Society of Echocardiography. J Am Soc Echocardiogr 2013;26:1013-32.

17. Lee MS, Finch W, Mahmud E. Cardiovascular complications of radiotherapy. Am J Cardiol 2013;112:1688-96.

Cite this article as: Hemu M, Chiang CJ, Bhatt PK, Ahmed A, Hein KZ, Mourad T, Randall ME, Palomo AP, Kramer JB, Fughhi I, Fogg L, Bonomi P, Okwuosa TM. Associations between sinus tachycardia and adverse cardiovascular outcomes and mortality in cancer patients. J Thorac Dis 2021;13(8):48454852. doi: $10.21037 /$ jtd-21-779
18. McBride W, Jackman JD Jr, Grayburn PA. Prevalence and clinical characteristics of a high cardiac output state in patients with multiple myeloma. Am J Med 1990;89:21-4.

19. Ali MK, Ewer MS. Cancer and the cardiopulmonary system. New York: Raven Press, 1984.

20. Reddy YNV, Melenovsky V, Redfield MM, et al. HighOutput Heart Failure: A 15-Year Experience. J Am Coll Cardiol 2016;68:473-82.

21. Lee DH, Park S, Lim SM, et al. Resting heart rate as a prognostic factor for mortality in patients with breast cancer. Breast Cancer Res Treat 2016;159:375-84.

22. Boudoulas H, Rittgers SE, Lewis RP, et al. Changes in diastolic time with various pharmacologic agents: implication for myocardial perfusion. Circulation 1979;60:164-9.

23. Opdahl A, Ambale Venkatesh B, Fernandes VRS, et al. Resting heart rate as predictor for left ventricular dysfunction and heart failure: MESA (Multi-Ethnic Study of Atherosclerosis). J Am Coll Cardiol 2014;63:1182-9.

24. Fox K, Ford I, Steg PG, et al. Heart rate as a prognostic risk factor in patients with coronary artery disease and left-ventricular systolic dysfunction (BEAUTIFUL): a subgroup analysis of a randomised controlled trial. Lancet 2008;372:817-21.

25. Swedberg K, Komajda M, Böhm M, et al. Ivabradine and outcomes in chronic heart failure (SHIFT): a randomised placebo-controlled study. Lancet 2010;376:875-85.

26. Effect of metoprolol CR/XL in chronic heart failure: Metoprolol CR/XL Randomised Intervention Trial in Congestive Heart Failure (MERIT-HF) Lancet 1999;353:2001-7.

27. Qdaisat A, Wu W, Lin JZ, et al. Clinical and CancerRelated Predictors for Venous Thromboembolism in Cancer Patients Presenting to the Emergency Department. J Emerg Med 2020;58:932-41.

28. Yeung SC, Habra MA, Thosani SN. Lung cancerinduced paraneoplastic syndromes. Curr Opin Pulm Med 2011;17:260-8. 